OPENING TALK 


\title{
Stellar black holes: Cosmic history and feedback at the dawn of the universe
}

\author{
I. Felix Mirabel ${ }^{1,2}$ \\ ${ }^{1}$ CEA-Saclay, IRFU/DSM/Service d'Astrophysique. 91191 Gif sur Yvette. France \\ ${ }^{2}$ IAFE-UBA-CONICET. cc 67, suc. 28. (C1428) Buenos Aires. Argentina \\ email: felix.mirabel@cea.fr
}

\begin{abstract}
Significant historic cosmic evolution for the formation rate of stellar black holes is inferred from current theoretical models of the evolution of massive stars, the multiple observations of compact stellar remnants in the near and distant universe, and the cosmic chemical evolution. The mean mass of stellar black holes, the fraction of black holes/neutron stars, and the fraction of black hole high mass X-ray binaries (BH-HMXBs)/solitary black holes increase with redshift. The energetic feedback from large populations of BH-HMXBs form in the first generations of star burst galaxies has been overlooked in most cosmological models of the reionization epoch of the universe. The powerful radiation, jets, and winds from BH-HMXBs heat the intergalactic medium over large volumes of space and keep it ionized until AGN take over. It is concluded that stellar black holes constrained the properties of the faintest galaxies at high redshifts. I present here the theoretical and observational grounds for the historic cosmic evolution of stellar black holes. Detailed calculations on their cosmic impact are presented elsewhere (Mirabel, Dijkstra, Laurent, Loeb, \& Pritchard 2011).
\end{abstract}

Keywords. Black holes, microquasars, cosmology

\section{The dark ages}

Motivated by a talk of Rashid Sunyaev at the 7th Microquasar Workshop, I became interested in exploring the possible role of black holes of stellar mass in the early cosmic evolution, and in particular, on how feedback from accretion of the remnants of massive stars could have affected the intergalactic medium during the dark ages. The so called "dark ages" of the universe began about 400,000 years after the Big Bang as matter cooled down and space became filled with neutral hydrogen for hundreds of millions years. How most of the matter in the universe became again ionized (reionized) in less than a billon year is a question of topical interest in cosmology. The recent detection of the explosion of a massive star (Salvaterra R. et al. 2009) at $z \sim 8.2$ and the observation of galaxies (Bouwens et al. 2010) up to $z \sim 8$ support the idea that the ultraviolet radiation from massive stars in the first galaxies played an important role in the reionization. However, from the luminosity density of the most distant galaxies it has been claimed that the UV flux available from massive stars may not have been enough to keep fully reionized the universe (Bouwens et al. 2010). Because X-rays have a longer mean free path than the ultraviolet photons, it is proposed that accreting stellar black holes provided heating and secondary ionizations over large volumes of space (Mirabel et al. 2011). 


\section{Formation of black holes by implosion: cosmic historic evolution of BH-HMXBs}

Theoretical models show that the evolution and final fate of massive stars strongly depend on the initial metallicity and rotation. Stars with low metal content and initial masses of a few tens of solar masses collapse directly as black holes, with no energetic supernova natal kicks (Heger et al. 2003; Meynet \& Maeder 2005). On the other hand, recent hydrodynamic simulations of the formation of stars with low metal content (Krumholz et al. 2009; Turk et al. 2009, Stacy et al. 2009) show that a substantial fraction of these stars form as small multiple systems dominated by binaries with typical masses of tens of solar masses. Therefore, from current theoretical models it is inferred that the majority of high mass stellar binaries of low metallicity should remain gravitationally bound after the implosion, ending as BH-HMXBs, which are known to be powerful sources of X-rays, massive winds, and relativistic jets (microquasars, see Mirabel \& Rodríguez 1999). In the context of these models and the cosmic evolution of metallicity and star formation evolution it is then expected that: 1) the mass of stellar black holes, 2) the fraction of black holes/neutron stars, and 3) the fraction of black hole binaries/solitary black holes should increase with redshift. Therefore, the formation rate of BH-HMXBs must have been significantly larger in the early universe than in later epochs.

\section{Formation rate of stellar black holes as function metallicity}

How massive stars evolve and die depends on their initial mass, metal content, angular momentum, and whether they are born in isolation or in multiple systems (Meynet \& Maeder 2005). Despite these complexities the expected cosmic evolution of BH-HMXBs mentioned above is consistent with the following observations of stellar black holes and neutron stars in the near and distant universe.

a) In agreement with the theoretical expectations (Heger et al. 2003, Meynet \& Maeder 2005), the masses of black holes in high mass x-ray binaries determined dynamically seem to be a decreasing function of the metallicity of the host galaxy (Crowther et al. 2010). The black holes in the high mass binaries M 33 X-7, NGC 300 X-1 and IC10 X-1 which are in small galaxies of low metallicity, have masses in the range of 16 to 30 solar masses, which are larger than the mass of any known stellar black hole in the Milky Way and Andromeda galaxies.

b) It has been proposed that most ultraluminous X-ray sources (ULXs) are BH-HMXBs that contain black holes of several tens of solar masses in low metallicity environments, accreting in a slightly critical regime (Zamperi \& Roberts 2009). It is found that the occurrence rate of ULXs per unit galaxy mass in nearby galaxies is a decreasing function of the mass of the host galaxy (Swartz et al. 2009), namely, of its metal content. One extreme case in the local universe is that of the metal-poor $\left(Z<0.05 Z_{\odot}\right)$ ring Cartwheel galaxy, where it is estimated (Mapelli et al. 2009) that more than $\sim 100$ stellar black holes of $30-80$ solar masses might have been generated via direct collapse (implosion) during the last $10^{7} \mathrm{yr}$. The X-ray luminosity of this small galaxy of low metallicity is $10^{42} \mathrm{erg} / \mathrm{s}$, which rivals that of low luminosity AGN.

c) The kinematics in three dimensions of Galactic black hole binaries relative to their birth place provides evidence for black hole formation by implosion. So far, the space kinematics has been determined for five Galactic black hole binaries. The kinematics of the three black holes that have masses equal or larger than 10 solar masses suggest that they have been form directly, without very energetic supernovae, whereas the two black holes with less than 10 solar masses were form with energetic natal explosions. In fact, the microquasars GRO J1655-40 and XTE 1118+48 which host respectively black holes 


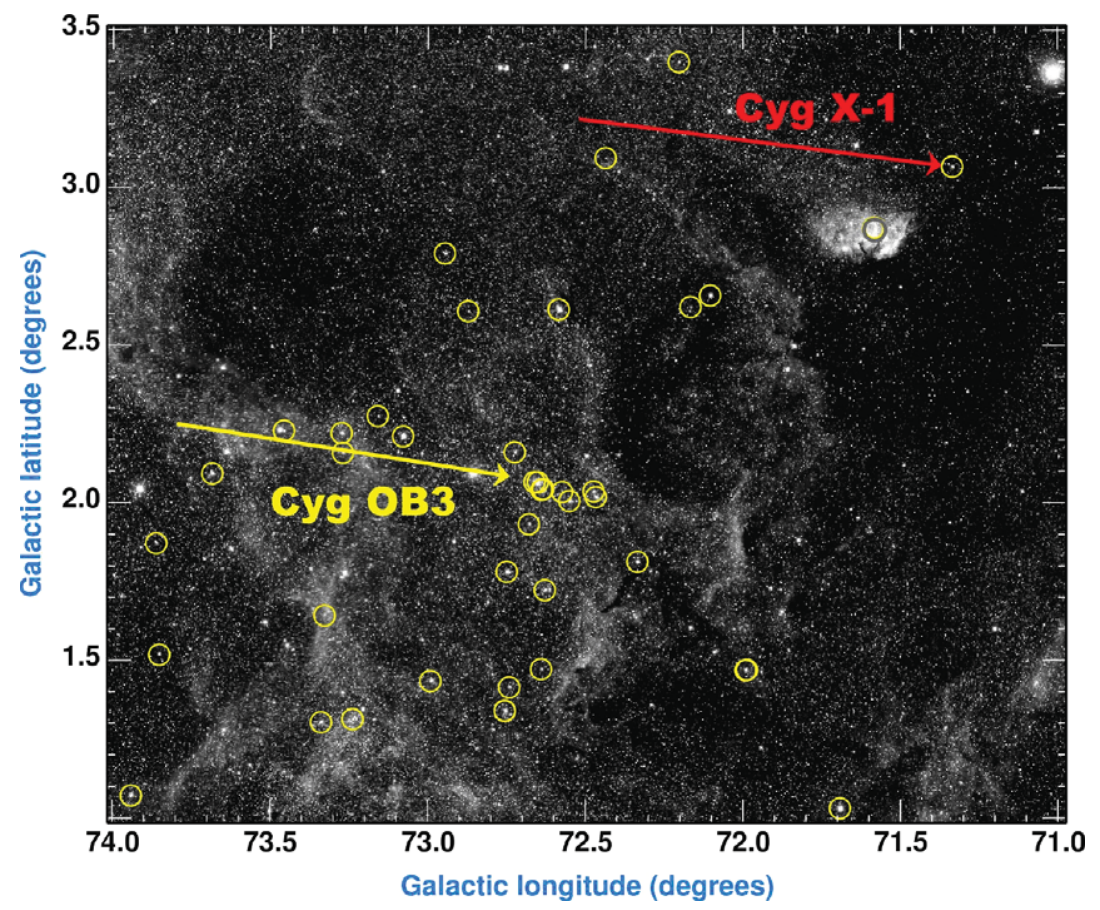

Figure 1. The kinematics represented in this optical image of the sky around the black hole x-ray binary Cygnus X-1 and the association of massive stars Cygnus OB3 shows that Cygnus X-1 remained anchored at its birth place in the association Cygnus OB3. The red arrow represents the motion in the sky of the radio counterpart of Cygnus X-1 for the past 0.5 million years. The yellow arrow the average Hipparcos motion of the massive stars of Cygnus OB3 (circled in yellow) for the past 0.5 million years. Despite the different observational techniques used to determine the proper motions, Cygnus X-1 moves in the sky as Cygnus OB3. At a distance of 2 $\mathrm{kpc}$, the space velocity of Cygnus X-1 relative to that of Cygnus OB3 is $<9 \mathrm{~km} / \mathrm{s}$, from which it is inferred that not more than one solar mass could have been suddenly ejected during the formation of the black hole in a natal supernova. From Mirabel, I.F. \& Rodrigues (2003).

of 5 and 7 solar masses have runaway space velocities relative to their environment of $112 \pm 18 \mathrm{~km} / \mathrm{s}$ and $177 \pm 33 \mathrm{~km} / \mathrm{s}$, respectively (Mirabel et al. 2002, Mirabel et al. 2001). On the contrary, Cyg X-1 which contains a black hole of 10 solar masses remained anchored at its birth place in the association Cyg OB3, and not more than one solar mass could have been suddenly ejected during a possible natal supernova (Mirabel \& Rodrigues, 2003).

The low mass X-ray binaries GRS 1915+105 and V404 Cyg that contain black holes of 14 and 12 solar masses respectively have both peculiar velocities relative to their environment of several tens of $\mathrm{km} / \mathrm{s}$ that lie on the plane of the Galaxy and are mostly in radial direction towards the Galactic Centre. However, their velocity components perpendicular to the Galactic plane are very small, $10 \pm 4 \mathrm{~km} / \mathrm{s}$ for GRS 1915+105 (Dhawan et al. 2007) and $0.2 \pm 3 \mathrm{~km} / \mathrm{s}$ for V404 Cyg (Miller-Jones et al. 2009). The kinematics of pulsars show that natal kicks have no preferential direction, and in this context the large peculiar velocities on the plane of the Galaxy of these two low mass X-ray binaries with black holes of more than 10 solar masses are most probably due to Galactic diffusion, rather than to energetic natal kicks.

d) The theoretical expectation that very massive stars with high metal content may end as neutron stars instead of black holes (Meynet \& Maeder 2005) is consistent with 
observations of some newly formed neutron stars. In fact, young neutron stars observed as Soft Gamma Ray Repeaters (SGRs) and Anomalous X-ray Pulsars (AXPs) are found in young clusters of massive stars. SGR 1806-20 (Mirabel et al. 2000, Figer et al. 2005) and AXP CXOU J1647-45 are associated with parent clusters of massive stars, the latter being inside the young cluster Westerlund 1 (Muno et al. 2006). These clusters are in the inner Galaxy and have metal contents larger than solar. Assuming coeval formation of the most massive stars in the clusters and the progenitors of the young neutron stars, lower limits of 40 and 50 solar masses for the masses of the progenitors of these neutron stars are inferred (Figer et al. 2005, Muno et al. 2006).

e) Gamma Ray Bursts of long duration (LGRBs) mark the formation of black holes by the collapse of massive stars. Although a fraction of dark GRBs may require local extinction columns of $A v>1 \mathrm{mag}$, the majority of the hosts of LGRBs are faint, irregular galaxies of limited chemical evolution (Le Floc'h et al. 2003, Fruchter et al. 2006). GRB 060505 and GRB 060614 were observed (Della Valle et al. 2006, Fynbo et al. 2006, GalYam et al. 2006) with no luminous SNe, and one possible explanation -among others (Gehrels et al. 2006)- is black hole formation by direct collapse.

f) A number of supernovae, classified as core collapse Type II show extremely low expansion velocity and an extraordinarily small amount of ${ }^{56} \mathrm{Ni}$ in the ejecta (Zampieri et al. 2003). These SNe are under-energetic with respect to a typical Type II supernova and may originate from the explosion of a massive progenitor in which the rate of early infall of stellar material on the collapsed core is large. Events of this type could form a black hole remnant, giving rise to significant fallback with late-time accretion and relatively small kicks. Recently it has been observed a low-energy core-collapse supernova without hydrogen envelope (Valenti et al. 2009), suggesting a link of faint supernovae with long-duration gamma-ray bursts.

g) There is increasing evidence for enhanced LGRB rate at $z>3$, as expected from the increase of specific star formation rate with decreasing metallicity (Daigne et al. 2006, Kistler et al. 2008, Qin et al. 2010).

\section{Feedback from accreting stellar black holes}

As in AGN, feedback from accreting stellar black holes is observed in the form of energetic radiation, powerful jets, and massive winds.

Energetic radiation: Super-Eddington radiation that last relatively short times is observed in outbursts of black hole novae, which have low-mass stellar donors (e.g. Nova Muscae, V404 Cyg). BH-HMXBs can exhibit a somewhat steady form of super-Eddington radiation of up to $10^{41} \mathrm{erg} / \mathrm{s}$. This is observed in the extragalactic ULXs, which often have spectra that resemble the very high (super-soft) state observed in some Galactic black hole binaries (e.g. GRS 1915+105). The majority of ULXs exhibit a complex curvature spectrum which can be modeled by a cool disk component together with a power law which breaks above $3 \mathrm{keV}$, probably due to a cool, optically thick corona produced by super-Eddington accretion flows (Gladstone et al. 2009).

On the other hand, the satellites Fermi and Agile detected gamma-ray flares from two high mass X-ray binaries: from the microquasar Cygnus X-3 and from Cygnus X-1. TeV flares may have been observed with the Cherenkov telescope MAGIC. This high energy emission from microquasars has been modeled in the context of jet leptonic and hadronic models (Vila \& Romero 2010, Romero 2008).

Powerful jets: Steady large-scale jets have been observed in several Galactic black hole binaries (e.g. 1E 1740.7-2942; GRS 1758-258; SS 433), which are viewed as small scale analogues of the extragalactic FR II radio galaxies. The existence of powerful 
super-Eddington jets of low radiation efficiency has been revealed by the multiwavelength observations of the HMXB SS 433, where the jets entrain atomic nuclei with velocities of $0.26 c$, have mechanical energies $>10^{39} \mathrm{erg} / \mathrm{s}$, and are capable of blowing laterally the nebula W50 up to distances of tens of parsecs. Large scale bow-shocked nebula produced by powerful dark jets have been observed in the BH-HMXB Cygnus X-1 (Gallo et al. 2005), and more recently in the HMXB S26 in the galaxy NGC 7793 (Pakull et al. 2010). The mechanical energy injected by S26 is $>10^{40} \mathrm{erg} / \mathrm{s}$, showing that the overall energy injected by these HMXB microquasars during their whole lifetime can be several orders of magnitude that of the photonic and baryonic energy from a typical core collapse supernova. Further evidence for powerful jets of low radiation efficiency has been obtained by the observations in the X-rays with Chandra of moving jets in the black hole binaries XTE J1550-564 (Corbel et al. 2002) \& H 1743-32 (Corbel et al. 2005). These observations show in real time the formation of double-lobe X-ray and radio lobes. The $\mathrm{X}$-rays are produced by synchrotron mechanism, implying that electrons are accelerated up to $\mathrm{TeV}$ energies by shocks in the moving lobes at parsec distances from the BHXRBs.

Massive winds: The connection between winds, jets and x-ray emission has been observed with unprecedented detail in GRS 1915-105 (Neilsen et al. 2011). The X-ray observations reveal ejections from the inner disk in the form of jets followed by winds from the outer disk that are massive enough to quench the jet and produces transitions in the X-ray overall output. Non-relativistic massive outflows in the form of winds of ionized and neutral gas have now been observed in accreting black holes of all mass scales.

\section{Discussion and results}

\section{Ionization and thermal history of the Intergalactic Medium}

Early star-forming galaxies as the ones recently discovered with the Hubble Space Telescope up to $z \sim 8$ (Bouwens et al. 2010), when the universe was only $~ 800$ millon years old, must have caused the reionization of the intergalactic medium (IGM). However, it is currently believed that the ultraviolet radiation from the massive stars in those galaxies was enough to produce and keep over large volumes of space most of the IGM reionized and heated to temperatures of $\sim 10^{4} \mathrm{~K}$ (Roberston et al. 2010). This belief resides in the assumption that the escape fraction into the IGM of UV photons $f_{\text {esc }}=0.1-0.2$ as in galaxies at $z \sim 3$. Furthermore, the recombination rate depends -besides on the hydrogen density- on the temperature of the IGM. Since the mean free path of the Xrays in a neutral medium is much larger than that of the UVs and X-rays are capable of producing multiple ionizations, Mirabel et al. (2011) propose that the X-ray radiation from the large population of BH-HMXBs in the firsts star burst galaxies ionized and heated to temperatures of $\sim 10^{4} \mathrm{~K}$ the IGM at large distances in the low density regions, bringing down the recombination rate, and therefore keeping the whole IGM ionized.

The idea that black holes may have played a role in the reionization era of the universe was developed previously by Madau et al. (2004). Motivated by the early reports from the Wilkinson Microwave Probe (WMAP) of a large optical depth to Thomson scattering that would have implied a very early reionization epoch, it was proposed a scenario where the universe was first reionized by intermediate black holes at $z>20$. However, a more accurate determination from WMAP3 later lead to a revision of the optical depth downward (Page et al. 2007), to a value consistent with reionization significantly later, at the epoch when the first stars were form. On the other hand, it has been shown (Alvarez et al. 2009, Milosavljevic et al. 2009) that feedback from accretion to solitary black holes significantly affects further inflow and the consequent injection of radiation 
and high energy particles to the surrounding medium. Therefore, if X-rays played a role in the heating and reionization of the Intergalactic Medium (IGM), in the context of the current simulations of the first generations of massive stars (Krumholz et al. 2009, Turk et al. 2009, Stacy et al. 2009), and the observations of stellar black holes in the near and distance universe, the most realistic alternative to quasi-radial, Bondi-like accretion would be accretion to black hole stellar remnants in the first generations of high mass binary stars, namely, BH-HMXBs.

On the other hand, current observational results indicate that galaxies -and therefore stellar black holes- were formed before supermassive black holes. In this context, heating of the IGM was first caused and maintained by accreting stellar black holes, before this role was taken over by AGN.

\section{Stellar black holes constrain the properties of dwarf galaxies}

The apparent disparity between the number of dwarf galaxies predicted by the Cold Dark Matter model of the universe, with the number of low mass galaxies observed so far in the halo of the Galaxy is a subject of topical interest in cosmology. Power et al. (2009) pointed out the possible implications of X-ray binaries in primordial globular clusters for the reionization, and therefore, galaxy formation at high redshifts.

As shown by Mirabel et al. (2011), once the IGM is heated to a temperature of $10^{4} \mathrm{~K}$, dark matter halos with masses below $10^{9} M_{\odot}$ no longer can accrete IGM material because the temperature of the infalling gas increases by an extra order of magnitude as its density increases on its way into these galaxies. In that regime, only gaseous halos with virial temperatures above $10^{5} \mathrm{~K}$ could have accreted fresh IGM gas and converted it to stars. The census of dwarf galaxy satellites of the Milky Way requires a related suppression in the abundance of low-mass galaxies relative to low-mass dark matter halos (Alvarez et al. 2009). The thermal history of the IGM therefore has a direct impact on the properties of the faintest galaxies at high redshifts as well as the smallest dwarf galaxies in the local universe.

It is interesting to note that black holes of different mass scales have a role in galaxy formation. Feedback from supermassive black holes halt star formation, quenching the unlimited mass growth of massive galaxies (Fabian 2009). Feedback from stellar black holes in HMXBs during the reionization epoch suppress the number of dwarf galaxies with masses $<10^{9} M_{\odot}$. Therefore, BH-HMXBs in the early universe are an important ingredient to reconcile the apparent disparity between the observed number of dwarf galaxies in the Galactic halo with the number of low mass galaxies predicted by the Cold Dark Matter model of the universe.

\section{References}

Alvarez, M. A. Wise, J. H., \& Abel, T. 2009, ApJ 701, L133

Bouwens, R. J. et al. 2010, ApJ 709, L16

Corbel, S. et al. 2002, Science 298, 196

Corbel, S. et al. 2005, ApJ, 632, 504

Crowther, P. A. et al. 2010, MNRAS, 403, L41

Daigne, F. \& Rossi, E. M., Mochkovitch, R. 2006, MNRAS 372, 1034

Della Valle, M. et al. 2006, Nature, 444, 1050

Dhawan, V., et al. 2007, ApJ 668, 430

Fabian, A. C. 2009, Proc. of IAU Symposium 267, B.M. Peterson, R.S. Somerville, \& T. StorchiBergmann, eds.

Figer, D. F. 2005, ApJ 622, L49

Fruchter, A. S. et al. 2006, Nature 441, 463 
Fynbo, J. P. U. et al. 2006, Nature 444, 1047

Gal-Yam, A. et al. 2006, Nature 444, 1053

Gallo, E. et al. 2005, Nature 436, 819

Gehrels, N. et al. 2006, Nature 444, 1044

Gladstone, J. C., Roberts, T. P., \& Done, C. 2009, MNRAS 397, 1836

Heger, A. et al. 2003, ApJ 591, 288

Kistler, M. D. et al. 2008, ApJ 673, L119

Krumholz, M. R. et al. 2009, Science 323, 754

Le Floc'h, E. et al. 2003, A\&A 400, 499

Madau, P. et al. 2004, ApJ 604, 484

Mapelli, M., Colpi, M., \& Zampieri, L. 2009, MNRAS 395, L71

Meynet, G. \& Maeder, A. 2005, $A \mathscr{E} A 429,581$

Miller-Jones, J. C. A. et al. 2009, ApJ 706, L230

Milosavljevic, M. et al. 2009, ApJ 698, 766

Mirabel, I. F. \& Rodríguez, L. F. 1999, Ann. Rev. Astron. Astrophys. 37, 409

Mirabel, I. F., Fuchs, Y., \& Chaty, S. 2000, AIP Conf. Proc. 526, 814

Mirabel, I. F. et al. 2001, Nature 413, 139

Mirabel, I. F. et al. 2002, A\&SA 395, 595

Mirabel, I. F. \& Rodrigues, I. 2003, Science 300, 1119

Mirabel, I. F., Dijkstra, M., Laurent, Ph., Loeb, A., \& Pritchard, J. R. 2011, submitted to A\&A Muno, M. P. et al. 2006, ApJ 636, L41

Neilsen, J., Lee, J. C., \& Remillard, R. 2011, Proc. of IAU Symposium 275, G. E. Romero, R. A. Sunyaev 85 T. Belloni, eds.

Page, L. et al. 2007, ApJ Supp. 170, 335

Pakull, M. W., Soria, R., \& Motch, C. 2010, Nature 466, 209

Power, C. et al. 2009, MNRAS 364, 1146

Qin, S. F. 2010, MNRAS 406, 558

Roberston, B. E. et al. 2010 Nature 468, 49

Romero, G. E. 2008, Rev. Mex. Astron. Astrof. 33, 82

Salvaterra, R. et al. 2009., Nature 461, 1258

Stacy, A., Greif, T. H., \& Bromm, V. 2010, MNRAS 403, 45

Swartz, D. A., Tennat, A. F., Soria, R. 2009, ApJ 703, 159

Turk, M. J., Abel, T., \& O'Shea, B. 2009, Science 325, 601

Valenti, S. et al. 2009, Nature 459, 674

Vila, G. S., \& Romero, G. E. 2010, MNRAS 403, 1457

Zampieri, L. et al. 2003, MNRAS 338, 711

Zamperi, L. \& Roberts, T. P. 2009, MNRAS 400, 677

\section{Discussion}

PE'ER: Where would you draw the line between jet (continuous) and "blobs" ejection as seen in GRS 1915; is there evidence for jet there at all?

MIRABEL: In GRS 1915+105 we have imaged with the VLBA a compact "continuous" jet long of tens of astronomical units and velocities $<0.2 c$ associated to the low-hard state. The sudden transitions from the low-hard state to the high soft state mark the onset of discrete and bright condensations or shocks that move away in the form of collimated jets at apparent superluminal motions.

KYLAFIS: In the slide that you showed about GRS 1915, the optical should appear also. Was it detected?

MirABEL: No, because GRS 1915 is on the Galactic plane at $>6 \mathrm{kpc}$ and there are about 30 magnitudes of optical absorption along the line of sight. 
SAmBRUnA: In AGN (and GRS 1915) disk winds are observed (see F. Tombesi contribution) that most likely contribute to feedbak on large scales.

MIRABEL: I fully agree. In fact, besides the observations of ionized gas with Chandra reported in this meeting by Neilsen et al., recent observations of Mrk 231 revealed massive outflows of molecular gas. The ions moving at $0.26 c$ in SS433 are from winds that have been entrained and accelerated by the jets.

KAWAI: Half of GRBs are so-called "dark" GRBs with no optical counterpart. They may be formed in metal-rich environments. Thus, low-metalicity is not an essential condition for GRB formation.

MirABel: In a recent study, Perley et al. conclude that the source of obscuring dust is local to the vicinity of the GRB progenitor and may be highly unevenly distributed within the host galaxy. In fact, my former PhD student Emeric Le Floc'h did not detect with Spitzer any of the host galaxies of dark GRBs, which implies that these are not globally speaking, highly dusty galaxies. The production of dust may occur rapidly in associations of massive stars and the GRB could take place after other massive stars have already enriched locally the interstellar medium.

FENDT: You have been introducing the "universal model for jet-disk coupling" for BH. Why should it not work for neutron stars? Central masses are similar, also the disks should be similar (\& simirlaly relativistic).

MiRABEL: It may work for neutron stars, white dwarfs, and young stars. But I prefer to leave a more detailed answer to this question to the speakers that will discuss this issue later in the week.

Rodríguez: To correct for these two failures of the Cold Dark Matter model, do you need stellar-mass or supermassive black holes, or both?

MiRABEL: Both. Supermassive black holes may account for the absence of masive cusps in the central regions of galaxies. Stellar black holes in the early universe would have heated the IGM to temperatures of about $10^{4} \mathrm{~K}$ which would have impeded the formation of large numbers of low-mass galaxies by baryonic accretion onto small haloes of dark matter.

NiSHIKAWA: What causes the time lag between infrared and radio emission in GRS $1915+105 ?$

MirABEL: The time delay is due to the fact that in an adiabaticaly expanding plasma cloud, the radiation becomes transparent to longer wavelenths at later times. 